\title{
ENSO components of the Atlantic multidecadal oscillation and their relation to North Atlantic interannual coastal sea level anomalies
}

\author{
J. Park and G. Dusek \\ NOAA Center for Operational Oceanographic Products and Services 1305 East West Highway, Silver Spring, MD, USA \\ Correspondence to: J. Park (joseph.park@noaa.gov)
}

Received: 1 November 2012 - Published in Ocean Sci. Discuss.: 30 November 2012

Revised: 26 April 2013 - Accepted: 13 May 2013 - Published: 10 June 2013

\begin{abstract}
The El Niño Southern Oscillation (ENSO) and the Atlantic Multidecadal Oscillation (AMO) are known to influence coastal water levels along the East Coast of the United States. By identifying empirical orthogonal functions (EOFs), which coherently contribute from the Multivariate ENSO Index (MEI) to the AMO index (AMOI), we characterize both the expression of ENSO in the unsmoothed AMOI, and coherent relationships between these indices and interannual sea level anomalies at six stations in the Gulf of Mexico and western North Atlantic. Within the ENSO band (2-7 yr periods) the total contribution of MEI to unsmoothed AMOI variability is $79 \%$. Cross correlation suggests that the MEI leads expression of the ENSO signature in the AMOI by six months, consistent with the mechanism of an atmospheric bridge. Within the ENSO band, essentially all of the coupling between the unsmoothed AMOI and sea level anomalies is the result of ENSO expression in the AMOI. At longer periods we find decadal components of sea level anomalies linked to the AMOI at three southern stations (Key West, Pensacola, Charleston), but not at the northern stations (Baltimore, Boston, Portland), with values of coherence ranging from 20 to $50 \%$. The coherence of MEI to coastal sea level anomalies has a different structure and is generally weaker than that of the ENSO expressed AMOI influence, suggesting distinct physical mechanisms are influencing sea level anomalies due to a direct ENSO teleconnection when compared to teleconnections based on ENSO expression in the AMOI. It is expected that applying this analysis to extremes of sea level anomalies will reveal additional influences.
\end{abstract}

\section{Introduction}

The El Niño Southern Oscillation (ENSO) has been identified as an important forcing on sea level anomalies of the western Pacific Ocean and the west coast of the United States (Sweet et al., 2009). Along the east coast of the United States storm surges have a positive correlation with the El Niño phase of ENSO where patterns of anomalously high sea levels are attributed to El Niño related changes in atmospheric pressure over the Gulf of Mexico and eastern Canada, and to the wind field over the continental shelf of the northeast United States (Sweet and Zervas, 2011). The Atlantic Multidecadal Oscillation (AMO) has received less attention in relation to sea level anomalies, but has been related to coherent sea surface height variability along the western boundary of the North Atlantic (Frankcombe and Dijkstra, 2009), to regional variability of coastal sea levels along the mid-Atlantic coast (Sallenger et al., 2012) and to extreme sea levels along the coast of Florida (Park et al., 2010).

The dominant period of leading empirical orthogonal functions (EOFs) of ENSO and AMO are roughly an order of magnitude different: $5.1 \mathrm{yr}$ for the leading mode of the Multivariate ENSO Index (MEI) and roughly $70 \mathrm{yr}$ for the leading mode of the AMO index (AMOI). The first EOF of the AMOI closely matches a ten-year (121 month) moving average of the unsmoothed AMOI and effectively ignores interannual processes, however, the unsmoothed AMOI exhibits amplitude modulations at periods of roughly 3 to $7 \mathrm{yr}$ (as discussed below and illustrated in Fig. 1).

That ENSO has a signature in the AMO is not surprising, and progress has been made relating the degree of this teleconnection. For example, Guan and Nigam (2009) analyzed twentieth century Atlantic sea surface temperature 

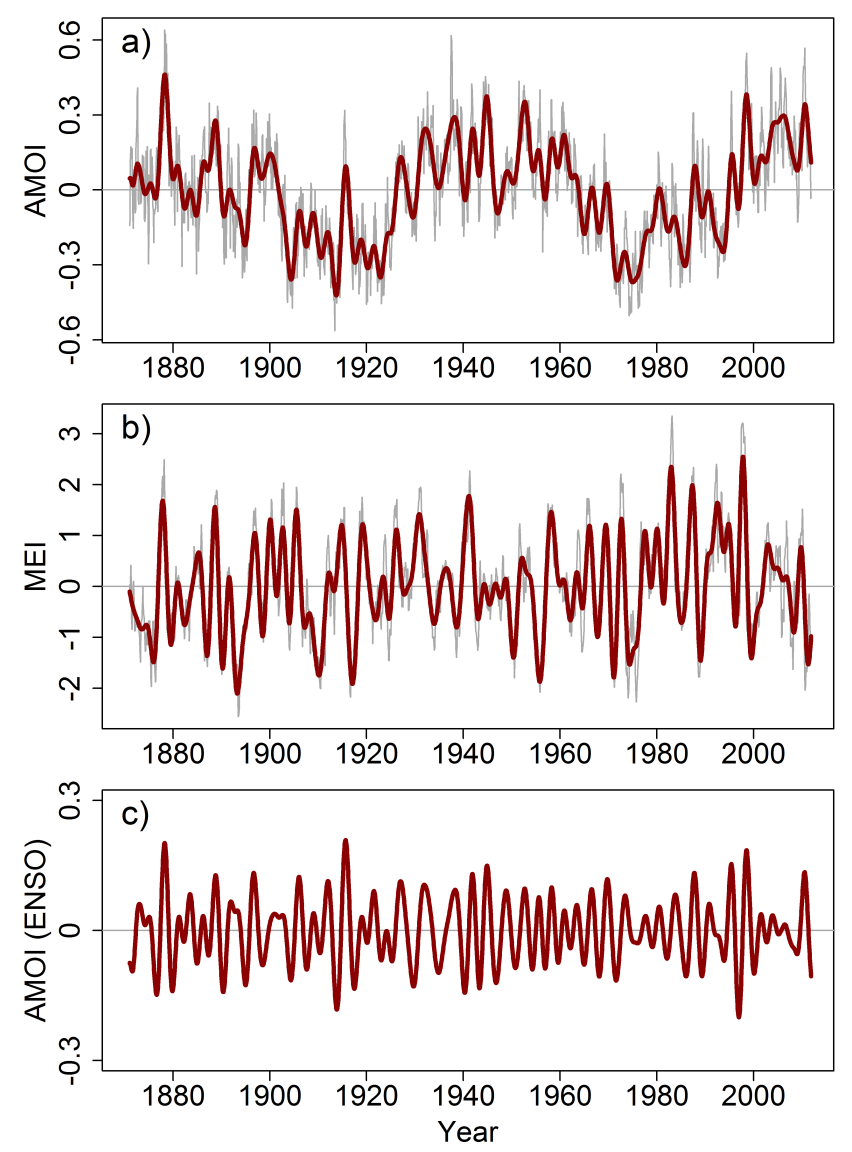

Fig. 1. Time series of: (a) unsmoothed AMOI (light gray) and reconstruction from the first 10 EOFs which include $76.2 \%$ of the variance (dark line), (b) MEI (light gray) and reconstruction of the first 10 EOFs which include $77.6 \%$ of the variance (dark line), and (c) reconstruction from the sum of AMOI(ENSO) EOFs with dominant frequencies in the ENSO band that transfer coherent power from MEI to AMOI. The horizontal axis is the calendar year.

(SST) variability while considering the influence of natural SST variability in the Pacific basin and the secular change in global SSTs. They found a significant influence of the Pacific basin on Atlantic SST variability: up to $45 \%$ of SST variance between Atlantic extratropical and tropical-subtropical basins in the conventional AMOI is due to this teleconnection.

The converse, that the AMO influences ENSO has also been suggested. Dong et al. (2006) found that a positive AMOI (warm phase) is associated with a deepened thermocline and reduced vertical stratification of the equatorial Pacific Ocean, which in turn leads to weakened ENSO variability. It is also apparent that ENSO and AMO act synergistically. For example, ENSO, AMO and the Pacific decadal oscillation (PDO) influence regional climate in the western United States, and depending on the relative phases of their indices manifest changes to wildfire potential (Schoennagel et al., 2007). Relative phases of ENSO and AMO combine to produce either favorable or unfavorable dynamic and thermodynamic factors influencing Caribbean basin tropical cyclone activity (Klotzbach, 2011). Further, interactions between high frequency (interannual) components represented by climate indices have been related to multidecadal hemispheric climate-regime shifts. These changes are characterized by transitions between distinct atmospheric and oceanic circulation patterns, and by altered ENSO variability (Wyatt et al., 2012).

It therefore seems reasonable to assume that geophysics encapsulated by the MEI and AMOI are interrelated with each other, constituting part of a feedback system between the two. The degree to which these forcings are expressed from one index to the other should be a valuable source of information for those analyzing and applying climate indices to correlations of geophysical variables and observations.

The purpose of this paper is twofold. First, to examine the extent of ENSO variability expressed in the unsmoothed AMOI, and second, to separate this influence in relation to coastal sea level variability in the Gulf of Mexico and the western North Atlantic. We first examine the joint spectral properties of the MEI and AMOI, identifying AMOI EOFs with ENSO frequencies that contribute coherently from MEI to AMOI. Isolation of these ENSO components within the AMOI allows for an estimate of the spectral power contributed to the AMOI by ENSO. We then examine the joint spectral properties of these AMOI ENSO components in relation to interannual sea level variability at six coastal stations from the Gulf of Mexico along the North Atlantic coast of North America.

\section{ENSO modes in the AMOI}

The data analyzed consist of monthly time series of two readily available climate indices, unsmoothed AMOI (NOAA ERSL, 2012; Fig. 1a) and the extended MEI (Wolter and Timlin, 2011; Fig. 1b). In Fig. 1 the unsmoothed index data are shown with thin gray lines and time series reconstructions using the first 10 EOFs are shown with thick lines. Ten EOFs were chosen based on a threshold of $75 \%$ total variance $(76.2 \%$ for the AMOI, $77.6 \%$ for MEI). EOFs are computed from singular spectrum analysis (Ghil et al., 2002), and the EOF reconstructions here serve simply as a visual guide. Salient features regarding discernable patterns of oscillation do not change much if several fewer or additional EOFs are included.

To quantify the extent to which ENSO is expressed in the AMOI, we use spectral coherence (squared coherence) to estimate the portion of coherent signal power that a single input - single output (SISO) linear system would transmit if MEI were the input and unsmoothed AMOI the output. In order for these spectral estimates to be approximately valid, the time series should be bandlimited and stationary. The bandlimited nature of these indices is guaranteed by the physical 
Table 1. AMOI(ENSO) EOFs with dominant frequencies in the ENSO band ( 2 to $7 \mathrm{yr}$ ) that contribute coherent power from the MEI to AMOI.

\begin{tabular}{lrrrrrr}
\hline EOF Rank & 5 & 6 & 7 & 8 & 9 & 10 \\
\hline Period (yr) & 3.51 & 3.60 & 5.14 & 4.97 & 2.82 & 2.57 \\
Variance (\%) & 3.1 & 3.0 & 2.9 & 2.7 & 2.4 & 2.2 \\
\hline
\end{tabular}

variables upon which they are computed, large-scale geophysical processes with inertia and damping that preclude step or delta function components. To assess stationarity, we applied the augmented Dickey-Fuller test to both the unsmoothed AMOI and MEI, and found the null hypothesis of a unit root in the characteristic equation for each time series rejected at the $99 \%$ confidence level.

To identify AMOI EOFs with coherent power between the MEI and AMOI, which we denote AMOI(ENSO) modes, we first select AMOI EOFs that have a dominant spectral peak in the ENSO band, resulting in seven modes with EOF eigenvalue ranks (based on decreasing partial variance) of 5 , $6,7,8,9,10$ and 11 . The next criteria quantifies the extent to which linear combinations of these modes contribute coherent power from the MEI to the AMOI. We evaluate this by maximizing the reduction in total coherence between the MEI and AMOI with sequential removal of all possible linear combinations of the seven AMOI EOFs with a dominant spectral peak in the ENSO band.

To estimate the fraction of coherent power reduction, $R$, we compute the ratio of the integral of coherence between the two models:

$$
R_{i}=1-\int \gamma_{\mathrm{MAE}}^{2}(i) \mathrm{d} f / \int \gamma_{\mathrm{MA}}^{2} \mathrm{~d} f
$$

where $\gamma_{\mathrm{MA}}^{2}$ is the coherence function of MEI to unsmoothed AMOI (Fig. 2, blue line), and $\gamma_{\mathrm{MAE}}^{2}(i)$ is the coherence function between MEI and the AMOI EOF reconstruction minus the $i$-th linear combination of the prospective AMOI(ENSO) modes. The limits of integration are the ENSO band periods from 2 to $7 \mathrm{yr}$. With seven prospective modes (EOFs 5 to 11) there are 127 combinations, and we select the combination of modes that maximizes the coherence reduction with the result that removal of the six AMOI EOFs 5, 6, 7, 8,9 , and 10 , produces a maximal reduction of $R=79.0 \%$ across the ENSO band. This indicates that EOF 11 (with a period of $2.25 \mathrm{yr}$ and comprising $2.1 \%$ of the AMOI variance) is not contributing coherently between the MEI and AMOI. We therefore identify the six modes listed in Table 1 as the AMOI(ENSO) modes.

Figure 1c plots a time series reconstruction from these six AMOI(ENSO) EOFs. Inspection of Fig. 1 reveals an uneven transmission of MEI variability to the AMOI(ENSO) reconstruction as a function of time. For example, around the year 1945 the MEI (Fig. 1b) presents relatively little variability,

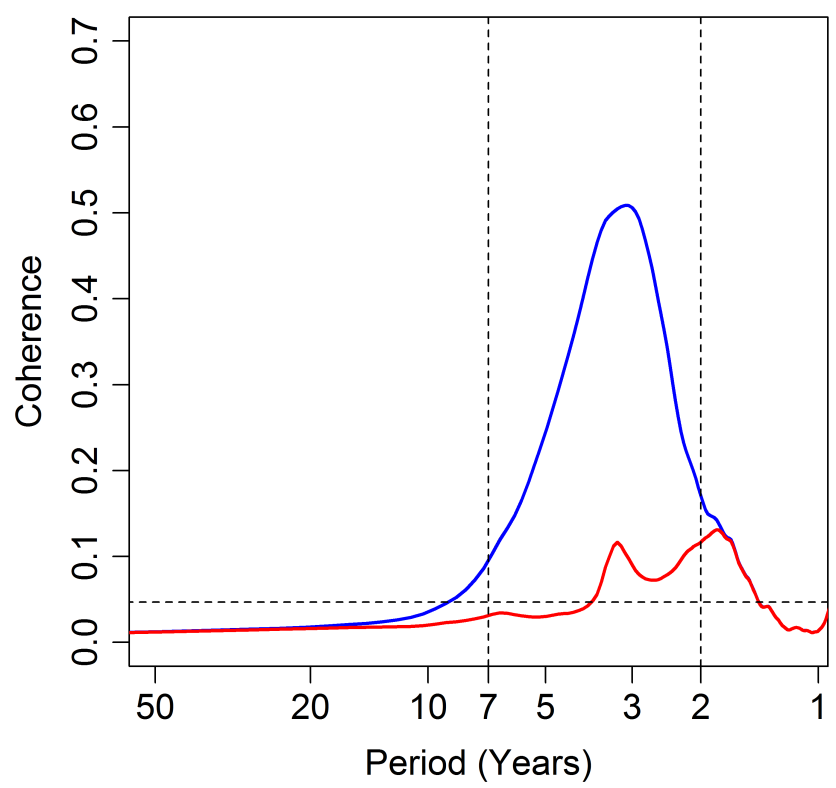

Fig. 2. Coherence of MEI to unsmoothed AMOI (blue), and MEI to AMOI reconstructed from all AMOI EOFs minus AMOI(ENSO) EOFs. The horizontal axis is the spectral period in years. Vertical dashed lines mark ENSO band frequencies, the horizontal dashed line quantifies the threshold to reject the null hypothesis of random coherence at the $95 \%$ confidence level (Thompson, 1979).

while the reconstruction of AMOI(ENSO) modes show large amplitude variations (Fig. 1c). At other times, one can perceive stronger amplitude correlation. This suggests that there is not a simple proportional relationship between the MEI and it's expression in the unsmoothed AMOI, rather one of some complexity.

A comparison of $\gamma_{\mathrm{MA}}^{2}$, the coherence of MEI to unsmoothed AMOI, with $\gamma_{\text {MAE }}^{2}(i)$, the coherence between MEI and the AMOI minus the AMOI(ENSO) modes, is shown in Fig. 2 with blue and red lines respectively. At discrete temporal periods between 3 to $4 \mathrm{yr}$, we find that up to $50 \%$ of AMOI variability in the ENSO band is coherent with the MEI. Outside the ENSO band there is little difference in relation to the full AMOI coherence, suggesting that the AMOI(ENSO) modes are not influencing AMOI variability at other timescales. The magnitude of the integrated reduction (79\%) indicates that over the period 1871-2011 (the analysis time period of the MEI and AMOI data) roughly three quarters of the SST variance expressed in the AMOI within the ENSO band is coherent with the MEI, suggesting that an ENSO teleconnection is physically responsible for this portion of the North Atlantic SST variance.

It may be worth remarking that from a time series analysis perspective (infinite bandwidth), the reduction in AMOI variance from removal of AMOI(ENSO) EOFs is $16.3 \%$ (sum of variance in Table 1). That the bandlimited coherent power reduction in the ENSO band is estimated at 
$79 \%$ implies that $0.790 \times 0.163=12.9 \%$ of the time series variance is attributed to the AMOI(ENSO) EOFs, while $16.3-12.9=3.4 \%$ are contributions from AMOI(ENSO) EOFs at frequencies outside the ENSO band.

Power spectral density estimates computed with smoothed periodograms of the three signals shown in Fig. 1 are presented in Fig. 3, and provide a basis for comparison with spectral characteristics of the sea level anomalies. As expected, the AMOI has the greatest power at multidecadal periods in excess of $20 \mathrm{yr}$. Interesting features include the band of enhanced variability around a period of $10 \mathrm{yr}$, and the smaller, smooth peaks between 3 and $6 \mathrm{yr}$ that are similar to features in the MEI spectrum. The MEI spectrum characteristically reveals peak power in the ENSO band, while the AMOI(ENSO) spectrum presents a prominent energy distribution across the ENSO band as well as a significant but low power component with an annual period. (This annual component is $40 \mathrm{~dB}$ below the ENSO band peak amplitude, a ratio of 1E-4.) Since the AMOI(ENSO) EOFs were selected only if their dominant spectral component was within the band from 2 to $7 \mathrm{yr}$, we conclude that this low amplitude annual component represents a linear superposition of low energy components from two or more of the six AMOI(ENSO) EOFs.

In addition to the mean spectral power conveyed from the MEI to the AMOI, the average temporal relationship between the two is of interest. Figure 4 plots the cross-correlation function between MEI and AMOI(ENSO) EOFs, and we find that MEI leads the expression of ENSO in the AMOI by an average of 6 months. Potential mechanisms for this correlation are discussed below.

\section{Interannual sea level anomalies}

Having suggested that ENSO influence on the AMOI can be estimated with spectral coherence, and in light of the fact that sea level anomalies in the western North Atlantic are influenced by both ENSO and AMO, we would like to probe the extent to which these influences can be separated in relation to western North Atlantic coastal sea level variability. We examine monthly mean sea level anomalies at six longterm tidal gauges in the Gulf of Mexico and along the eastern seaboard of the United States (Fig. 5). Table 2 lists the stations along with the period of record for each station, and Fig. 6 plots the anomalies computed from monthly mean sea levels with the average seasonal cycle and linear sea level trend removed (Zervas, 2009; NOAA CO-OPS, 2012).

To establish perspective on the spectral distribution of these anomalies, power spectral density estimates for each of the time series in Fig. 6 are shown in Fig. 7. All stations are dominated by variance at the decadal and longer timescales, with interannual and ENSO band power components also apparent. However, it is difficult to identify clear attributes in spectral variance with respect to either ENSO or
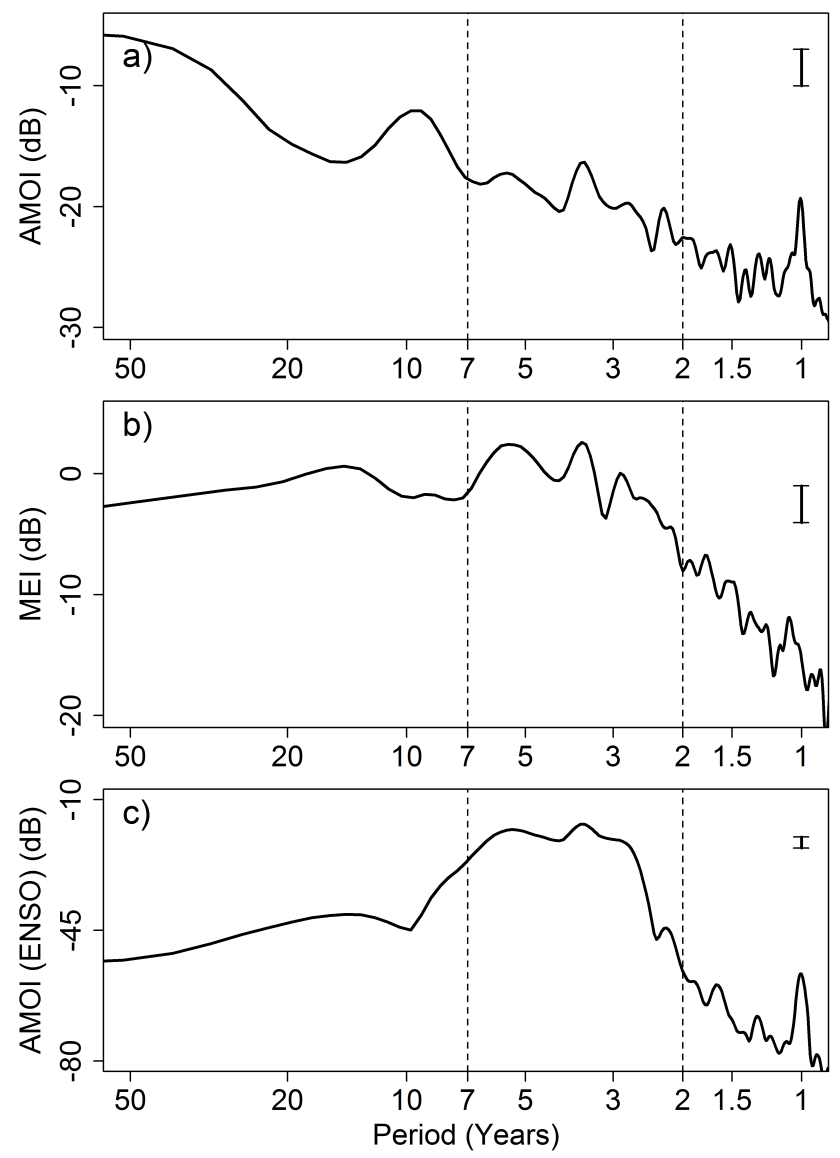

Fig. 3. Power spectral density estimates of (a) unsmoothed AMOI, (b) MEI, and (c) reconstruction of the sum of the six AMOI EOFs with dominant frequencies in the ENSO band that contribute coherently from MEI to AMOI. The horizontal axis is the spectral period in years. Vertical dashed lines mark ENSO band frequencies, vertical bar quantifies the $95 \%$ confidence interval.

Table 2. Tidal stations and period of record.

\begin{tabular}{ll}
\hline Station & Period of record \\
\hline Pensacola, FL & January 1924-December 2011 \\
Key West, FL & January 1913-December 2011 \\
Charleston, SC & January 1922-December 2011 \\
Baltimore, MD & January 1903-December 2011 \\
Boston, MA & January 1921-December 2011 \\
Portland, ME & January 1912-December 2011 \\
\hline
\end{tabular}

AMO forcings. We will again use a SISO spectral coherence model to estimate the relative forcings between MEI, AMOI and AMOI(ENSO) as inputs, and the sea level anomalies as output.

Coherence between the unsmoothed AMOI and sea level anomalies are presented in Fig. 8 with blue lines, and between reconstructions based on AMOI(ENSO) EOFs and sea level anomalies in red. The horizontal dashed line quantifies 


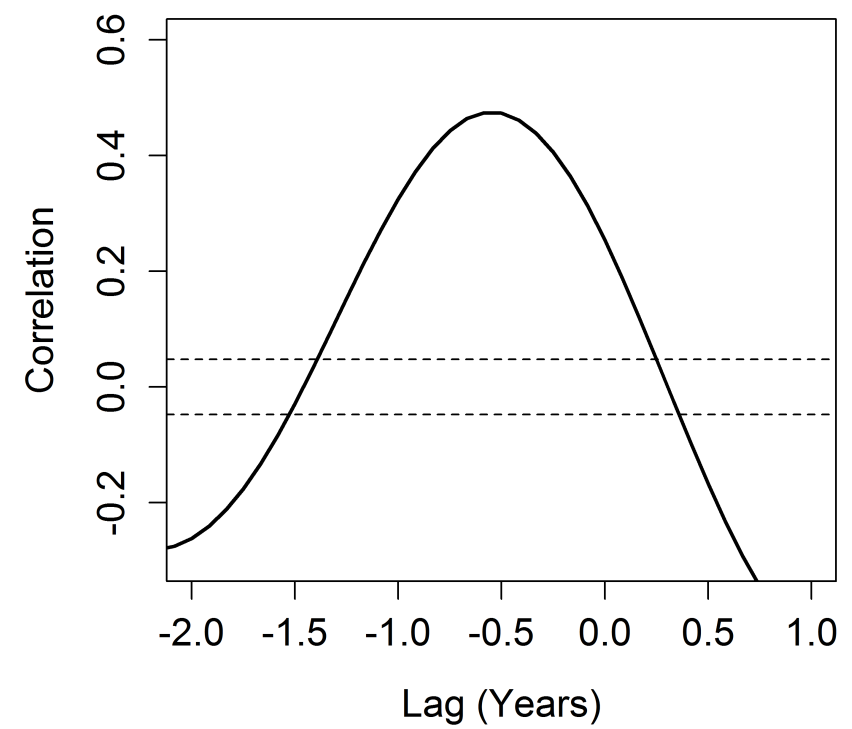

Fig. 4. Cross correlation between MEI and reconstruction from the six AMOI(ENSO) EOFs. The peak correlation value is 0.47 with MEI leading AMOI by 6 months. Dashed lines quantify $95 \%$ confidence intervals outside of which the null hypothesis of zero correlation is rejected.

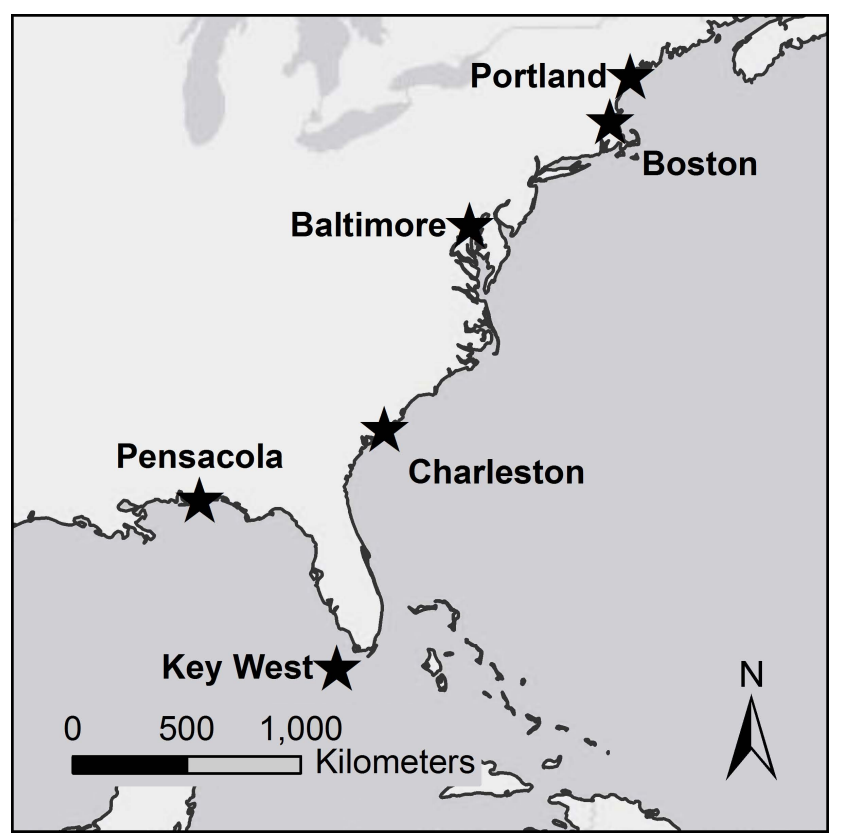

Fig. 5. Map of the six tidal stations.

the threshold to reject the null hypothesis of random coherence at the $95 \%$ confidence level (Thompson, 1979). At periods from 6 to $15 \mathrm{yr}$, with a peak near $9 \mathrm{yr}$, there is evidence of weak AMOI coupling at Key West, Pensacola and Charleston. Estimates of the coherence reduction between AMOI and AMOI(ENSO) forcings integrated over periods from 6 to $15 \mathrm{yr}$ are $86.8,85.9$ and $97.9 \%$ respectively. These
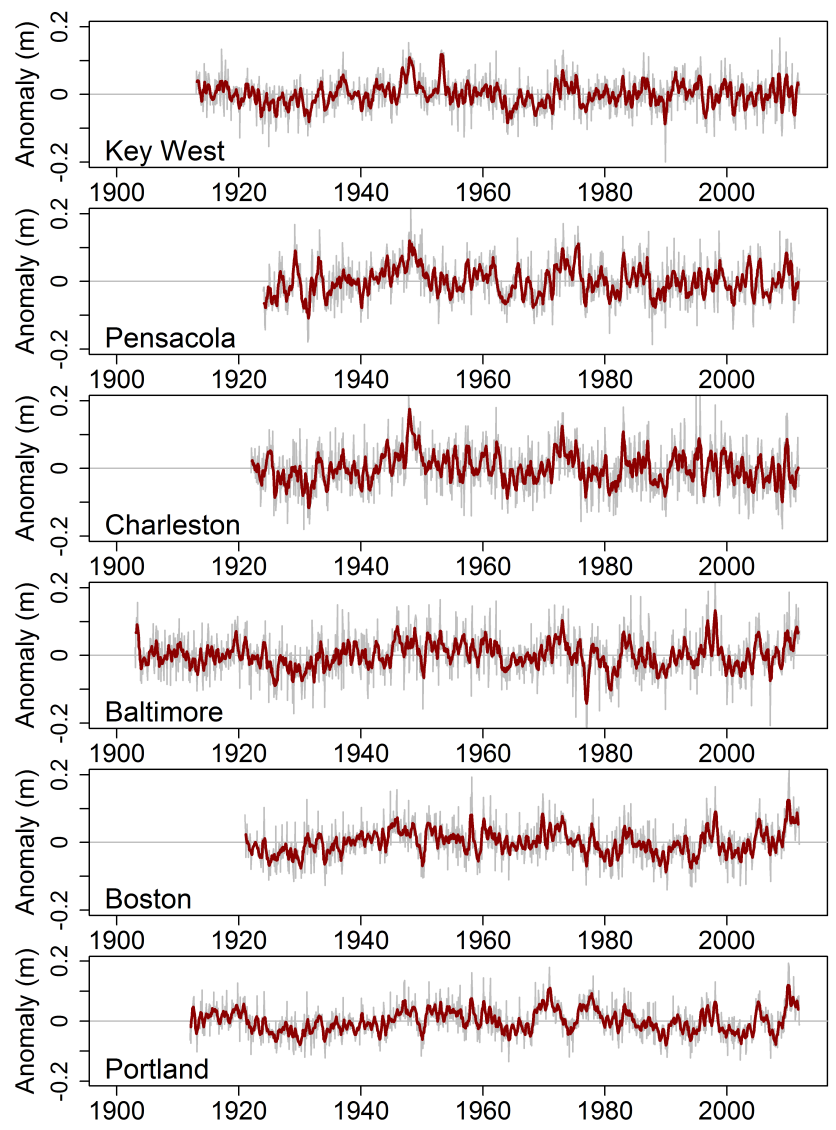

Fig. 6. Interannual variation of monthly mean sea level (gray line) at six long-term tidal stations along the east coast of the United States. The average seasonal cycle and linear sea level trend have been removed. A six month moving average is shown with the thick line. The horizontal axis is the calendar year.

large reductions indicate that mechanisms related to Atlantic SST (as expressed in the AMOI), which are not being influenced by ENSO, are coherent with decadal variability of sea level anomalies at these three southeastern US stations. Within the limitations of a SISO model, the estimate of peak coherence at these stations is roughly 0.2 at Key West and Pensacola, and 0.3 at Charleston, suggesting that the fraction of sea level anomaly variance at a period of $9 \mathrm{yr}$ driven by non-ENSO forcings expressed in the AMOI are between 20 to $30 \%$.

Moving to periods from 4 to $2.6 \mathrm{yr}$, we observe broadband coherence centered at $2.8 \mathrm{yr}$ at Charleston, and a coupling centered at $3.2 \mathrm{yr}$ at Boston and Portland, although the Boston forcing is less distinct than at Portland. Coherence at all three stations is essentially equivalent between the AMOI and AMOI(ENSO) forcings of sea level anomalies, leading to the conclusion that over this frequency band sea level anomalies coherent with the AMOI are also coherent with the MEI, which is being expressed in AMOI. Thus we 


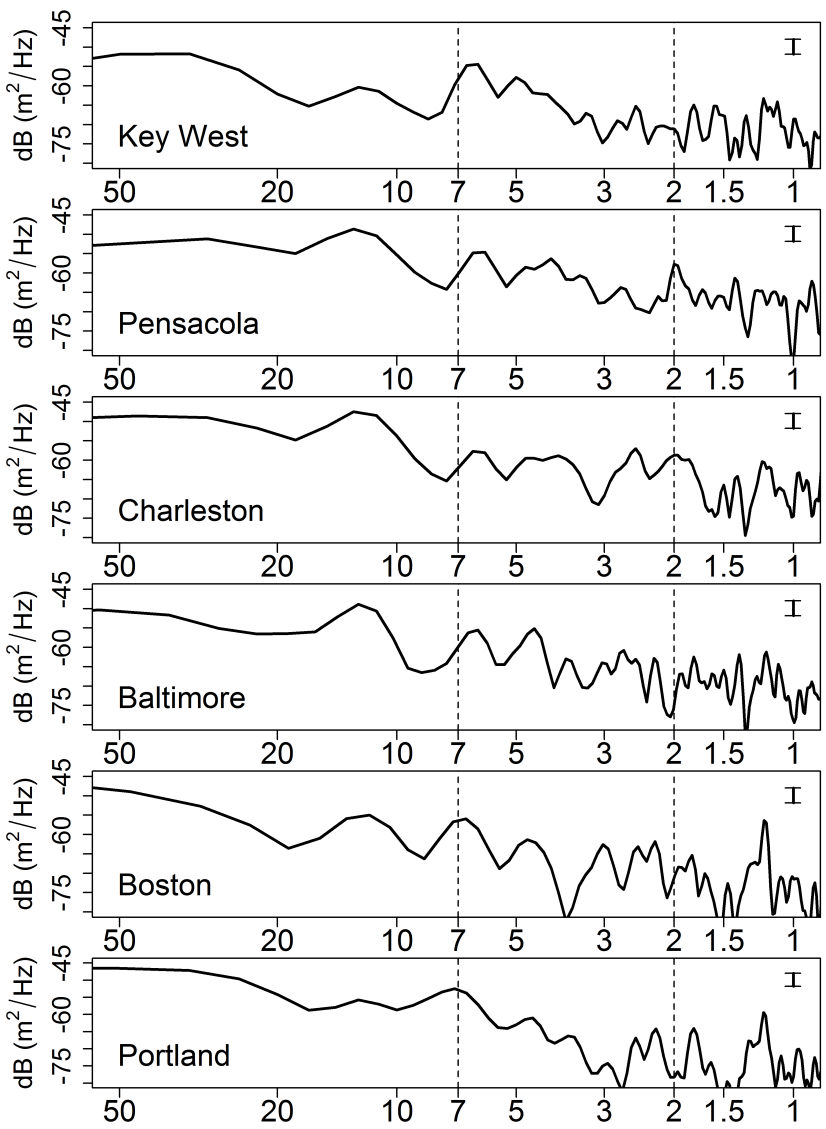

Fig. 7. Power spectral density estimates of interannual sea level variations at six long-term tidal stations along the east coast of the United States. The horizontal axis is the spectral period in years. Vertical dashed lines mark ENSO band frequencies, vertical bar quantifies the $95 \%$ confidence interval.

conjecture that ENSO forcings expressed in the AMOI are the drivers of this variability.

The next frequency band of interest covers periods from 2.6 to $1.8 \mathrm{yr}$, for which there are prominent peaks centered at $2.2 \mathrm{yr}$ at Portland, Boston and Baltimore, with less distinct couplings exhibited at Charleston and Pensacola. Each of these couplings shows nearly equivalent coherence whether AMOI or AMOI(ENSO) forcings are considered, again revealing sea level anomalies at these stations coherent with AMOI are also coherent with ENSO modes expressed in the AMOI. The estimate of coherence at the $2.2 \mathrm{yr}$ period peak is 0.4 at Baltimore and Portland, and 0.5 at Boston, indicating that 40 to $50 \%$ of the anomaly variance at this period is being forced by a mechanism expressed in the AMOI but due to ENSO forcing.

The final band with generally common features among the stations covers periods from 1.8 to $1.3 \mathrm{yr}$, with a peak coherence near $1.5 \mathrm{yr}$. Here, we find conflicting results in the sense that at Key West, Pensacola, Baltimore and Portland the AMOI coupling to sea level anomalies is stronger
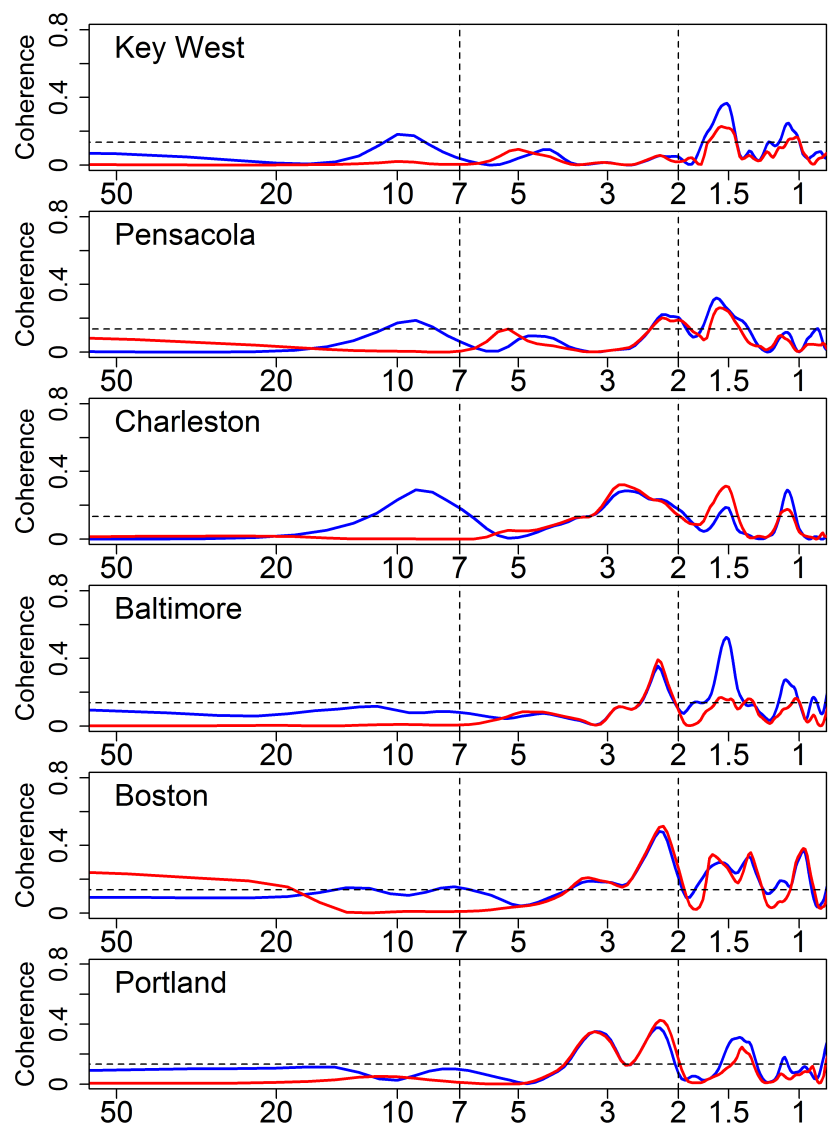

Fig. 8. Coherence between AMOI and interannual sea level variations (blue), and coherence between the reconstruction from the AMOI(ENSO) and interannual sea level variations (red). The horizontal axis is the spectral period in years. Vertical dashed lines mark ENSO band frequencies, the horizontal dashed line quantifies the threshold to reject the null hypothesis of random coherence at the $95 \%$ confidence level (Thompson, 1979).

than the AMOI(ENSO) expression, while at Charleston the $\mathrm{AMOI}(\mathrm{ENSO})$ is stronger, and at Boston the coherence is roughly equivalent. We will discuss these conflicts below.

In addition to AMOI and AMOI(ENSO) couplings to sea level anomalies, we also examine direct ENSO influence by estimating MEI to sea level anomaly coherence as shown in Fig. 9 (green line). For comparison, coherence between AMOI(ENSO) EOFs and the anomalies are shown in red (same curve as Fig. 8). The most prominent feature is the MEI to sea level anomaly coherence at Key West centered on a period of $7 \mathrm{yr}$, although the peak coherence is weak at 0.26 . Since coherence between AMOI(ENSO) and the sea level anomaly response is near zero (red line), the analysis suggests this component of sea level variability is coupled to ENSO forcings that are not expressed in the AMOI, for example, atmospheric pressure changes not reflected in North Atlantic SST. 

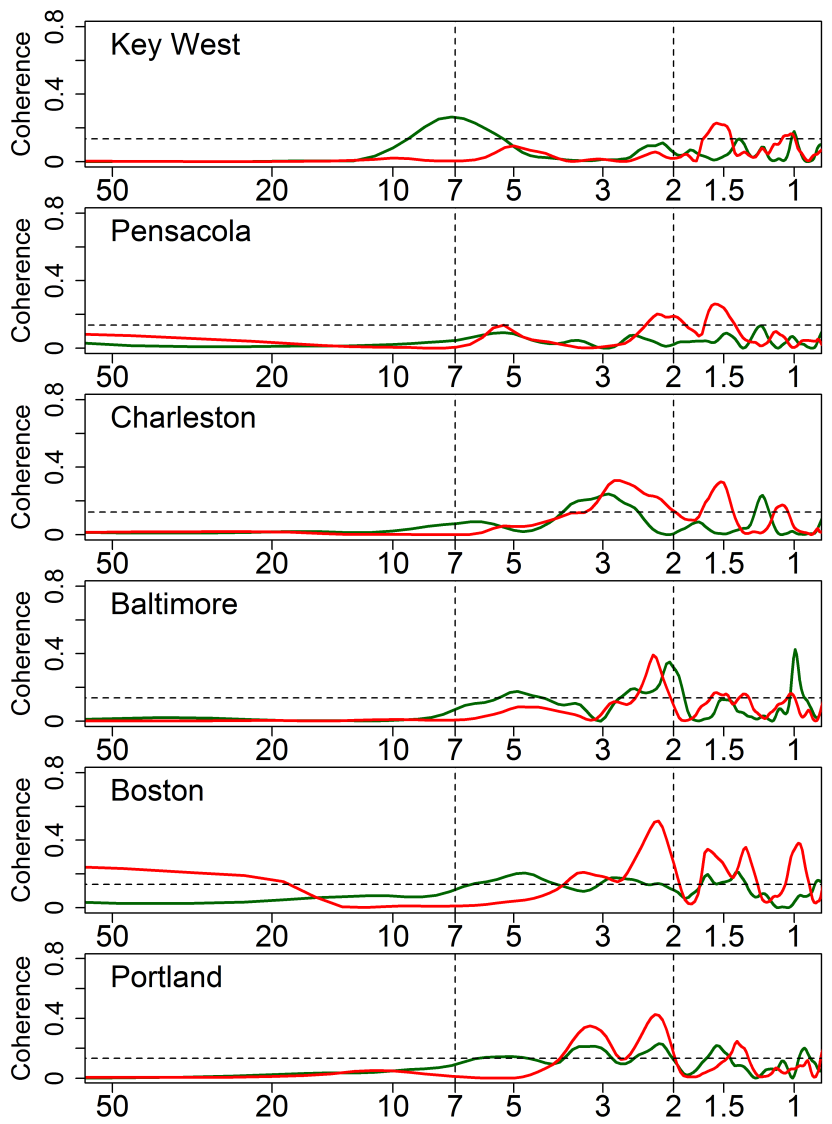

Fig. 9. Coherence between MEI and interannual sea level variations (green), and coherence between the reconstruction from AMOI(ENSO) EOFs and interannual sea level variations (red). The horizontal axis is the spectral period in years. Vertical dashed lines mark ENSO band frequencies, the horizontal dashed line quantifies the threshold to reject the null hypothesis of random coherence at the $95 \%$ confidence level (Thompson, 1979).

At Pensacola there is no evidence of MEI coupling to sea level anomalies, and in general the other stations exhibit weak coherence across the ENSO band. To the extent that these weak forcings have physical significance, the observation that the spectral shape and location of peaks between the MEI coherent (green) and AMOI(ENSO) coherent (red) curves do not generally coincide in frequency suggests that there are distinct geophysical forcings at work. Again, one might speculate that directly coupled MEI modes are influenced by ENSO driven atmospheric teleconnections, which are not expressed in the AMOI, while the AMOI(ENSO) related anomalies are coupled to ENSO forcings, which are expressed in Atlantic SST variability.

It is worth recalling that previous analysis of ENSO-forced sea level anomalies in the western North Atlantic found couplings between ENSO and storm surges, the extreme values of sea level anomalies (Sweet and Zervas, 2011). Thus we expect more significant results would be obtained in relation to MEI influence on the extreme sea level anomalies rather than on the anomalies themselves.

\section{Discussion}

When viewed within the constraints of a SISO linear systems model 40 to $50 \%$ of unsmoothed AMOI variability is coherent with the MEI at periods between 2.5 and $4 \mathrm{yr}$ (Fig. 2). By examining AMOI EOFs with dominant spectral frequencies in the ENSO band and which contribute coherently from the MEI to AMOI, we identified six AMOI EOFs that express Atlantic SST variance in response to ENSO related forcing. When these six AMOI(ENSO) EOFs are removed from the AMOI reconstruction, there is a $79 \%$ reduction in total coherent power between the MEI and AMOI (integrated over periods from 2 to $7 \mathrm{yr}$ ). Thus we are led to the conclusion that ENSO influences are expressed with some vigor in the unsmoothed AMOI, consistent with the findings of Guan and Nigam (2009). To the extent that the SISO model captures teleconnections between ENSO and Atlantic SST variability, one might expect a similar magnitude of expression in ENSO forced Atlantic SST geophysical dynamics, that over the ENSO band roughly three fourths of the total variance expressed in Atlantic SST forced dynamics are forced by ENSO variability.

In the time domain, cross correlation between the MEI and reconstruction of the AMOI(ENSO) EOFs finds MEI leading expression in the AMOI with a 6 month lead time (Fig. 4). This is consistent with a six month lag reported by GamizFortis et al. (2011) between single EOFs of North Atlantic SST and the Bivariate ENSO index with $3.6 \mathrm{yr}$ oscillatory period, as well as with lag times characteristic of an ENSO atmospheric bridge (Klein et al., 1999). Thus we expect the dominant forcing expressed in AMOI within the ENSO band corresponds to anomalies in atmospheric circulation associated with ENSO, which induce changes in the evaporation and cloud cover that, in turn, alter the net heat flux entering the North Atlantic (Klein et al., 1999).

Given that ENSO and AMO influence coastal sea level anomalies of the western North Atlantic (Sweet and Zervas, 2011; Frankcombe and Dijkstra, 2009; Park et al., 2010), and having identified ENSO forced modes of the AMOI, one can separate influences from ENSO forced AMOI; AMOI; and MEI to these anomalies. Concerning AMOI coupling without ENSO forcing, we find evidence that Atlantic SST variability as expressed in the unsmoothed AMOI is partially coherent with coastal sea level anomalies in a broadband centered on a period near $9 \mathrm{yr}$ at the three southern stations of Key West, Pensacola and Charleston. The peak strength of this forcing varies from 20 to $30 \%$, and we note that this energy is apparent in the power spectral estimate of the unsmoothed AMOI (Fig. 3). Since Pensacola is located within the northern Gulf of Mexico it should not be directly influenced by Atlantic meridonal overturning 
circulation (AMOC), which has been linked to AMO variability (Zhang, 2008), or by changes in coastal sea levels attributed to geostrophic relaxation of Gulf Stream transport (Noble and Gelfenbaum, 1992). Therefore, one can speculate that this coupling reflects an atmospheric teleconnection expressed in the Atlantic SST, which is coherent with coastal sea level anomalies at these three stations. Arguez et al. (2009) observed modes in both AMOI and the North Atlantic Oscillation (NAO) index with periods of approximately $10 \mathrm{yr}$, suggesting a component of decadal variability in North Atlantic SST from atmospheric forcing. However, their analysis finds maximal correlation between NAO and North Atlantic SST anomalies from North Carolina to Nova Scotia, with essentially no link south of Charleston. Since the $9 \mathrm{yr}$ modes coherent between AMOI and sea level anomalies shown in Fig. 8 are found only at the three southern stations, and not at the mid-Atlantic and northern stations, we suspect that the NAO is not a causative mechanism for these decadal sea level anomalies.

Regarding ENSO coupled Atlantic SST variability influencing coastal sea level anomalies, coherence between AMOI(ENSO) EOFs and anomalies at the six stations support two main conclusions. First, within the ENSO band (periods of 2 to $7 \mathrm{yr}$ ) essentially all of the forcing identified between unsmoothed AMOI and sea level anomalies are the result of ENSO expression in the AMOI. This can be seen in the overlap of the unsmoothed AMOI and AMOI(ENSO) coherence functions within the ENSO band (Fig. 8). Second, three broadband couplings centered at periods of 3.2, 2.8 and $2.2 \mathrm{yr}$ are presented in varying strength across the six stations. Specifically, the $3.2 \mathrm{yr}$ forcing is evidenced at Boston and Portland, the $2.8 \mathrm{yr}$ mode at Charleston, and the $2.2 \mathrm{yr}$ coupling at all stations except Key West.

At periods shorter than the ENSO band, persistent coupling is found to some degree at all stations near a period of $1.5 \mathrm{yr}$. This coupling is not consistently represented as being forced by either AMOI or AMOI(ENSO). It seems likely that the SISO model fails to account for influences other than ENSO or Atlantic SST variability at this period. For example, coastal sea levels at a specific station reflect oceanographic and climatological forcings not captured in the MEI or AMOI. Another potential influence is barometric effects and associated climatological changes related to storms as expressed in the NAO index, which has been correlated with western North Atlantic interannaul sea levels (Papadopoulos and Tsimplis, 2006). It is also known that changes in volume transport of the Gulf Stream impact the geostrophic balance of coastal sea levels along the western Atlantic on seasonal and interannual timescales (Noble and Gelfenbaum, 1992). Additionally, recent work by Wyatt et al. (2012) finds that shorter-term, interannual to interdecadal climate variability alters character according to polarity of their "stadium wave" hemispheric interaction, and suggest that mutual interaction between shorter-term variability and the stadium wave im- pacts interannual and multidecadal variability within the Atlantic sector.

When we examine direct MEI to sea level anomaly coherence, the coupling is generally weak and in most cases expressed with different spectral shapes than for either the direct AMOI or AMOI(ENSO) influences. We therefore speculate that there are distinct physical mechanisms driving the sea level anomalies coherent with the AMOI(ENSO) modes from those coherent directly with MEI. That the MEI couplings are weaker than those of the AMOI(ENSO) modes hints that ENSO influenced teleconnections that are related to SST variance have a stronger coupling to North Atlantic sea level anomalies than ENSO teleconnections not related to SST. We also note that analysis based on the extreme values of the sea level anomalies (storm surges) is likely to find a stronger influence from direct MEI coupling (Sweet and Zervas, 2011).

As noted by Wyatt et al. (2012), advantages of analyzing climate indices rather than unsmoothed observational variables include increased dynamical interpretability, increased signal-to-noise ratio and statistical significance, albeit at the expense of phenomenological completeness. This decoupling from raw fields limits our capability to engage in speculation regarding geophysical connections for the observed forcings. Further, the links we identified are limited to a few geospatial locations responding to synoptic-scale forcings captured by the indices. Clarification of the structure of these links and their attribution will involve the application of global climate models that capture the coupled ocean-atmosphere geophysical feedbacks.

\section{Conclusion}

We have assumed that a linear systems transfer function exists between the MEI and AMOI, and argued that since these indices are bandlimited and quasi-stationary, the spectral coherence provides a first-order estimate of coupling between the MEI and AMOI. However, if there is a physically based transfer function between these two indices it is likely nonlinear and further, it is nearly certain that a single inputsingle output model is insufficient to capture the inherent dynamics. Therefore, work remains to further explore these issues and clarify the decomposition of teleconnections between the two. Nonetheless, we believe that these results provide progress in that direction.

Identification of unsmoothed AMOI EOFs with dominant spectral periods in the ENSO band that contribute coherently from the MEI to AMOI allows us to isolate ENSO modes expressed in the AMOI. The results indicate that about three quarters $(79 \%)$ of the total coherent signal power in the ENSO band of the unsmoothed AMOI is attributed to forcing from the MEI, while couplings in the range of 40 to $50 \%$ can be found at discrete periods between 2.5 to $4 \mathrm{yr}$. From a temporal perspective, we find that the MEI forcing leads 
the unsmoothed AMOI response by an average of 6 months, characteristic of an ENSO atmospheric bridge.

Comparison of coherence spectra between AMOI(ENSO) EOFs and monthly sea level anomalies to coherence between unsmoothed AMOI and sea level anomalies, allows us to separate influences of ENSO coupled Atlantic SST anomalies from those that are not ENSO related. We find that three southern stations (Key West, Pensacola and Charleston) exhibit an AMOI to sea level anomaly response centered near a period of $9 \mathrm{yr}$, that ENSO forcings are not expressed in these AMOI influenced variations, and they do not seem consistent with NAO coupling. Within the ENSO band nearly all of the sea level anomaly variations that are coherent with AMOI are coherent with AMOI(ENSO) modes, accounting for 20 to $50 \%$ of the sea level anomaly variance. This suggests that ENSO forcing is acting through an atmospheric bridge teleconnection that is expressed in the unsmoothed AMOI, and it is the ENSO influence that is related to the sea level anomalies. These couplings are distinct from those between the MEI, which is not expressed in the AMOI and sea level anomalies, leading one to suspect distinct physical mechanisms are involved.

Edited by: D. Stevens

\section{References}

Arguez, A., O'Brien, J. J., and Smith, S. R.: Air temperature impacts over Eastern North America and Europe associated with low-frequency North Atlantic SST variability, Int. J. Climatol., 29, 1-10, 2009.

Dong, B., Sutton, R. T., and Scaife, A. A.: Multidecadal modulation of El Niño-Southern Oscillation (ENSO) variance by Atlantic Ocean sea surface temperatures, Geophys. Res. Lett., 33, L08705, doi:10.1029/2006GL025766, 2006.

Frankcombe L. M. and Dijkstra, H. A.: Coherent multidecadal variability in North Atlantic sea level, Geophys. Res. Lett., 36, L15604, doi:10.1029/2009GL039455, 2009.

Gamiz-Fortis, S. R., Esteban-Parra, M. J., Pozo-Vazquez, D., and Castro-Diez, Y.: Variability of the monthly European temperature and its association with the Atlantic sea-surface temperature from interannual to multidecadal scales, Int. J. Climatol. 31, 2115-2140, 2011.

Guan, B. and Nigam, S.: Analysis of Atlantic SST Variability Factoring Interbasin Links and the Secular Trend: Clarified Structure of the Atlantic Multidecadal Oscillation, J. Climate, 22, 42284240, doi:10.1175/2009JCLI2921.1, 2009.

Ghil, M., Allen, R. M., Dettinger, M. D., Ide, K., Kondrashov, D., Mann, M. E., Robertson, A. W., Saunders, A., Tian, Y., Varadi, F., and Yiou, P.: Advanced spectral methods for climatic time series, Rev. Geophys., 40, 3.1-3.41, doi:10.1029/2000RG000092, 2002.

Klein, S. A., Soden, B. J., and Lau, N.: Remote Sea Surface Temperature Variations during ENSO: Evidence for a Tropical Atmospheric Bridge, J. Climate, 12, 917-932, 1999.

Klotzbach, P. J.: El Niño-Southern Oscillation's Impact on Atlantic Basin Hurricanes and U.S. Landfalls, J. Climate, 24, 1252-1263, 2011.
NOAA CO-OPS: Center for Operational Oceanographic Products and Services, Sea Levels Online, available at: http:// tidesandcurrents.noaa.gov/sltrends/, (last access: 30 June 2012), 2012.

NOAA ESRL: Earth Systems Research Laboratory, Climate Time series AMO (Atlantic Multidecadal Oscillation) Index, AMO unsmoothed from the Kaplan SST V2, www.esrl.noaa.gov/psd/ data/timeseries/AMO/, (last access: 20 June 2012), 2012.

Noble, M. A. and Gelfenbaum, G. R.: Seasonal Fluctuations in Sea Level on the South Carolina Shelf and Their Relationship to the Gulf Stream, J. Geophys. Res., 97, 9521-9529, doi:10.1029/92JC00811, 1992.

Papadopoulos, A. and Tsimplis, M. N.: Coherent Coastal Sea-Level Variability at Interdecadal and Interannual Scales from Tide Gauges, J. Coastal Res., 22, 625-639, 2006.

Park, J., Obeysekera, J., and Barnes, J.: Temporal energy partitions of Florida extreme sea level events as a function of Atlantic multidecadal oscillation, Ocean Sci., 6, 587-593, doi:10.5194/os-6587-2010, 2010.

Sallenger, A. H., Doran, K. S., and Howd, P. A.: Hotspot of accelerated sea-level rise on the Atlantic coast of North America, Nature Clim. Change, 2, 884-888, doi:10.1038/nclimate1597, 2012.

Schoennagel, T., Veblen, T. T., Kulakowski, D., and Holz, A.: Multidecadal Climate Variability and Climate Interactions Affect Subalpine Fire Occurrence, Western Colorado (USA), Ecology, 88, 2891-2902, 2007.

Sweet, W. V. and Zervas, C.: Cool-Season Sea Level Anomalies and Storm Surges along the U.S. East Coast: Climatology and Comparison with the 2009/10 El Niño, Mon. Weather Rev., 139, 2290-2299, doi:10.1175/MWR-D-10-05043.1, 2011.

Sweet, W. V., Zervas, C., and Gill, S.: Elevated East Coast Sea Level Anomaly: June-July 2009. NOAA Technical Report NOS CO-OPS 051, August 2009. National Ocean and Atmospheric Administration, Center for Operational Oceanographic Products and Services, Silver Spring MD, http://tidesandcurrents.noaa. gov/publications/EastCoastSeaLevelAnomaly_2009.pdf, 2009.

Thompson, R. O. R. Y.: Coherence significance levels, J. Atmos. Sci., 36, 2020-2021, 1979.

Wolter, K. and Timlin, M. S.: El Niño/Southern Oscillation behaviour since 1871 as diagnosed in an extended multivariate ENSO index (MEI.ext), Int. J. Climatol., 31, 1074-1087, 2011.

Wyatt, M., Kravtsov, S., and Tsonis, A.: Atlantic Multidecadal Oscillation and Northern Hemisphere's climate variability, Clim Dynam., 38, 929-949, doi:10.1007/s00382-011-1071-8, 2012.

Zervas, C.: Sea Level Variations of the United States 18542006. NOAA Technical Report NOS CO-OPS 053, December 2009, National Ocean and Atmospheric Administration, Center for Operational Oceanographic Products and Services, Silver Spring MD, http://tidesandcurrents.noaa.gov/publications/Tech_ rpt_53.pdf, 2009.

Zhang, R.: Coherent surface-subsurface fingerprint of the Atlantic meridional overturning circulation, Geophys. Res. Lett., 35, L20705, doi:10.1029/2008GL035463, 2008. 